\title{
Perioperative Care in Patients with Ehlers Danlos Syndromes
}

\author{
Pradeep Chopra ${ }^{*}$, Linda Bluestein ${ }^{2}$ \\ ${ }^{1}$ Brown Medical School, Pain Management Center, Rhode Island, USA \\ ${ }^{2}$ Medical College of Wisconsin, Central Wisconsin, Wisconsin, USA \\ Email: *painri@yahoo.com
}

How to cite this paper: Chopra, P. and Bluestein, L. (2020) Perioperative Care in Patients with Ehlers Danlos Syndromes. Open Journal of Anesthesiology, 10, 13-29. https://doi.org/10.4236/ojanes.2020.101002

Received: December 7, 2019

Accepted: December 28, 2019

Published: December 31, 2019

Copyright $\odot 2020$ by author(s) and Scientific Research Publishing Inc. This work is licensed under the Creative Commons Attribution International License (CC BY 4.0).

http://creativecommons.org/licenses/by/4.0/ (c) (i) Open Access

\begin{abstract}
Background: There is an increasing recognition of patients with Ehlers Danlos Syndromes. The laxity of the ligaments and the weakness of the connective tissue has resulted in increasing number of patients requiring surgical intervention. Ehlers Danlos Syndromes are not about hypermobile joints only, they are associated with multiple co-existing conditions such as Chiari malformation, Tethered Cord Syndrome, spinal instability, abdominal pain, Dysautonomia and Mast Cell Activation Syndrome. The combined incidence of Ehlers Danlos Syndromes is 1 in 5000 people. Most experts believe that the actual incidence is much higher. Many of these cases are under-diagnosed. Nevertheless, patients with Ehlers Danlos Syndromes, diagnosed or undiagnosed often require surgical intervention. This review article has been written to shed light on the need for special consideration during anesthesia. Objectives: Our objective was to conduct a review of anesthetic considerations in patients with Ehlers Danlos Syndromes. Study Design: We used a narrative review design. Methods: This review was done using searches of PubMed, MEDLINE/OVID, SCOPUS, and manual searches of the bibliographies of known primary and review articles from inception to 2019. Other data sources included hand searches of publications driven by manuscript authors. Search terms included concepts of "Ehlers Danlos Syndrome", "EDS", "pain", "anesthesia", "surgery" and combination of terms. Search method was not restricted to any one language. Results: Articles were screened by title, abstract, and full article review. They were then analyzed by specific clinical indications and appropriate data was presented based on critical analysis of those articles. Limitations: More studies about the effect of anesthetic techniques and Ehlers Danlos Syndromes are required. Conclusions: Patients with Ehlers Danlos Syndromes may present with an array of coexisting medical conditions such as Dysautonomia, Mast Cell Activation Syndrome, Chiari Malformation, Tethered Cord Syndrome, Craniocervical instability, Gastropare-
\end{abstract}


sis, altered sensitivity to local anesthetics. Anesthetic techniques need to be modified according to the presentation. Preoperatively, a note should be made of the joints that sublux most often, craniocervical instability. They are prone to temporomandibular joint subluxation, obstructive sleep apnea and tracheomalacia, spontaneous pneumothorax, POTS, MCAS, insensitivity to local anesthetics and coagulation disorders. Intraoperative considerations should include fiberoptic intubation, IV fluid loading for POTS, avoid drugs that release histamine, unpredictable response to opioids, precautions for gastroparesis. Patient should be positioned to avoid joint subluxation and over stretching nerves. Postoperative considerations include maintaining hemodynamic stability, avoid exacerbation of symptoms of MCAS, high suspicion of occult bleeding, high risk of post dural puncture headache in patients undergoing spinal procedure or neuroaxial block.

\section{Keywords}

Anesthesia, Ehlers Danlos Syndromes, POTS, MCAS, Mast Cell

Activation Syndrome, Postural Orthostatic Tachycardia Syndrome

\section{Introduction}

This comprehensive review of perioperative care in patients with Ehlers Danlos Syndromes focuses on current understanding of anesthetic needs of this special population. As our understanding of Ehlers Danlos Syndromes has grown exponentially so has the need to understand perioperative management of these cases. There is a rising recognition of patients with Ehlers Danlos Syndromes. The laxity of the ligaments and the weakness of the connective tissue has resulted in increasing number of patients requiring surgical intervention. Ehlers Danlos Syndromes are not about hypermobile joints only, they are associated with multiple co-existing conditions such as Chiari malformation, Tethered Cord Syndrome, spinal instability, abdominal pain, Dysautonomia and Mast Cell Activation Syndrome. The combined incidence of Ehlers Danlos Syndromes is 1 in 5000 people [1]. Most experts believe that the actual incidence is much higher. Many of these cases are under-diagnosed. Nevertheless, patients with Ehlers Danlos Syndromes, diagnosed or undiagnosed often require surgical intervention. This review article has been written to shed light on the need for special consideration during anesthesia.

The Ehlers-Danlos Syndromes (EDS) are heritable connective tissue disorders characterized by joint hypermobility, skin hyperextensibility and connective tissue fragility. The 2017 International Classification for the Ehlers-Danlos Syndromes recognized 13 subtypes (Table 1). The diagnosis of EDS is based on a set of major and minor clinical criteria. A definitive diagnosis for all subtypes (except hypermobile type) depends on a molecular confirmation with identification of the causative variant(s) in the respective gene. As the genetic basis of hypermobile EDS subtype is unknown, its diagnosis relies on a strict set of clini- 
cal findings. In 2017, updated and much more strict criteria were established for the Hypermobile type of Ehlers-Danlos Syndrome (hEDS), by far the most common subtype of the Ehlers-Danlos Syndromes. At this same time, a new diagnostic category, Hypermobility Spectrum Disorders (HSD), is a group of conditions related to joint hypermobility $(\mathrm{JH})$. HSD are intended to be diagnosed after other possible diagnoses are excluded, such as any of the subtypes of Ehlers Danlos Syndromes (EDS) [2].

Table 1. Considerations for dealing with patients with EDS.

\begin{tabular}{|c|c|}
\hline \multirow{11}{*}{ Head and neck (6) } & Chiari malformation \\
\hline & Brain stem compression \\
\hline & Idiopathic intracranial hypertension \\
\hline & Atlantoaxial instability \\
\hline & Craniocervical instability \\
\hline & Epilepsy \\
\hline & Intracranial aneurysm \\
\hline & Temporomandibular joint dysfunction (7) \\
\hline & Headaches and migraines $(8)$ \\
\hline & Intermittent compression of vertebral arteries \\
\hline & Compression of upper cervical nerve roots from C0 to C2 hypermobility (6)) \\
\hline \multirow{5}{*}{ Spine (6) } & Segmental kyphosis and instability \\
\hline & Tethered cord syndrome \\
\hline & Tarlov cyst syndrome (8) \\
\hline & Meningeal ecstasias/cysts \\
\hline & Spontaneous cerebrospinal leak (9) \\
\hline \multirow{4}{*}{ Cardiovascular } & Structural defects such as mitral valve prolapse and aortic root dilatation (hEDS) $(11,17,18)$ \\
\hline & Dysautonomia (10) \\
\hline & Increased peripheral pooling (12) \\
\hline & Severe progressive cardiac-valvular aortic valve and mitral valve problems (cvEDS) \\
\hline \multirow{5}{*}{ Pulmonary } & Tracheomalacia \\
\hline & Rib subluxations \\
\hline & Pulmonary bullae \\
\hline & Decreased pulmonary volumes secondary to kyphoscoliosis \\
\hline & Obstructive sleep apnea (13) \\
\hline \multirow{6}{*}{ GI (19) } & Gastroparesis \\
\hline & Intestinal dysmotility (14) \\
\hline & Visceroptosis (15) \\
\hline & Hollow visceral (intestines, uterus) rupture \\
\hline & Rectal/uterine prolapse (16) \\
\hline & Visceral fragility (dEDS and vEDS) \\
\hline \multirow{2}{*}{ Urinary } & Neurogenic bladder $(6,17)$ \\
\hline & Interstitial cystitis $(20)$ \\
\hline \multirow{5}{*}{ Musculoskeletal (8) } & Joint subluxations and dislocations (8) \\
\hline & Myopathy (25) \\
\hline & Fatigue (22) \\
\hline & Poor joint proprioception (23) \\
\hline & Muscle weakness, axonal polyneuropathy, atrophy of muscles of hands and feet (24) \\
\hline \multirow{3}{*}{ Hematologic } & Bleeding disorders $(20)$ \\
\hline & Mast cell activation syndrome $(20,21)$ \\
\hline & Vascular fragility \\
\hline \multirow{6}{*}{ Neurological } & Dysautonomia (22) \\
\hline & Postural orthostatic tachycardia syndrome $(22)$, \\
\hline & Neuropathy $(26,27)$ \\
\hline & Small fiber peripheral neuropathy (28) \\
\hline & Entrapment neuropathy $(24,27,28)$ \\
\hline & Unpredictable response to local anesthetic \\
\hline
\end{tabular}


Patients with EDS present for surgical procedures frequently due to their tissue fragility, joint instability, vascular fragility, and association with neurosurgical co-existing conditions. This article will discuss principles of perioperative care in patients undergoing major or minor surgery.

\section{Methods}

A literature search was conducted using the main electronic databases including the Cochrane library, PubMed, EMBASE. The search terms used were, "Ehlers Danlos Syndrome”, "EDS”, “pain”, “anesthesia”, "surgery”. The papers reviewed were case series, case-controlled studies, review articles and observational studies. Given the paucity of information on preoperative management in EDS, any information obtained from literature relevant to EDS patients was screened. Since no information from laboratory animal or clinical studies was used, no approval from ethical committees was required (Table 2).

A search of online databases retrieved 180 articles. A majority of the papers were case reports. There was one paper with recommendations for perioperative management published in 2014 in Orphanet Journal of Rare diseases [3]. The case reports and case series focused more on vEDS comprises only $4 \%$ of patients with EDS [4] and obstetrical anesthesia. However, they were helpful in contributing and reinforcing the information on preoperative care from other sources. There was a set of obstetrical guidelines published in 2005 [5]. The medical community's experiences and understanding of EDS have expanded significantly since 2005 .

\section{Discussion}

This article has been divided into preoperative, intraoperative and postoperative recommendations for patients with EDS undergoing surgery. These are general

Table 2. Flow Diagram for sytematic review of records for anesthesia and EDS .

\begin{tabular}{|c|c|}
\hline $\begin{array}{l}\text { Records identified through database } \\
\text { searching }(\mathrm{n}=180)\end{array}$ & $\begin{array}{l}\text { Additional records identified through } \\
\text { other sources }(n=22)\end{array}$ \\
\hline$\downarrow$ & $\downarrow$ \\
\hline \multicolumn{2}{|c|}{ Records after duplicates were removed $(n=175)$} \\
\hline$\downarrow$ & $\downarrow$ \\
\hline Records screened $(n=175)$ & Records excluded on screening $(n=45)$ \\
\hline \multicolumn{2}{|l|}{$\downarrow$} \\
\hline $\begin{array}{l}\text { Full Text articles assessed for eligibility } \\
\qquad(\mathrm{n}=130)\end{array}$ & $\begin{array}{l}\text { Full Text articles excluded with reasons } \\
\qquad(\mathrm{n}=45)\end{array}$ \\
\hline \multicolumn{2}{|l|}{$\downarrow$} \\
\hline $\begin{array}{l}\text { Studies included in the descriptive } \\
\text { analysis }(n=130)\end{array}$ & \\
\hline
\end{tabular}


recommendations for the most common subtypes (hEDS and cEDS). There are some additional special considerations for vEDS. Many of these recommendations would also be appropriate to consider for patients with diagnosed or suspected Hypermobility Spectrum Disorder (people with symptoms related to joint hypermobility but do not meet the 2017 more strict diagnostic criteria for hEDS).

It should be noted that there can be crossover between subtypes (patients with one subtype of EDS can present with features of a different subtype). For example, a patient with hEDS may still present with some of the risks of vEDS such as vascular fragility and risk of hollow viscera rupture. Some of the issues that should be kept in mind when dealing with patients with EDS are listed in Table 1.

\section{Preoperative}

It is important to understand which subtype of EDS the patient has as well as have an awareness of the occasional possibility of overlap in pathologic features between the different subtypes. For example, a patient with cEDS may have features of vEDS such as aortic root dilatation or a vascular anomaly.

In a preoperative history and physical examination, it is important to note which joints are prone to recurrent subluxations or dislocations. Patients will have some joints that sublux more easily than others. These joints need to be protected by keeping them in the anatomical position during the intraoperative and postoperative periods. Additionally, positioning changes need to be carefully planned due to the potential for trauma to neural structures as well as any weak connective tissue such as ligaments, tendons, joint capsules etc.

Patients with EDS can have Craniocervical instability (CCI) with related deformation of the brainstem and upper spinal cord, basilar invagination or ventral brainstem compression. They can also have Chiari malformation with restricted CSF flow, Idiopathic Intracranial Hypertension, C4-C5 osteochondrosis and mid-thoracic osteochondrosis.

This population is also prone to Temporomandibular Joint Dysfunction (TMJ) [6]. Younger patients have a greater incidence of subluxations, whereas older patients develop restricted range of motion. With recurrent subluxations, the temporomandibular joint (TMJ) has a tendency to become arthritic, further restricting range of motion [7] [8]. Any of these conditions should lead one to strongly consider fiberoptic intubation in order to avoid manipulation of the cervical spine and minimize the risk of TMJ injury.

Obstructive sleep apnea has been reported in patients with EDS [9]. Patients with a history of stridor on expiration may have tracheomalacia. On palpation of the suprasternal notch, the trachea may feel soft.

A history of spontaneous pneumothorax or bullae on chest x-ray should be elicited. Rib subluxations are common in EDS and should be considered in the differential diagnosis of chest pain. If the patient has significant kyphosis and/or scoliosis pulmonary function testing should be considered. The level of the ky- 
phosis or scoliosis should be noted in case a neuraxial block is planned. Patients with EDS usually have a dynamic scoliosis.

Dysautonomia is a common comorbid condition in EDS [10] [11]. Postural orthostatic tachycardia syndrome (POTS) is the most common type of dysautonomia in the EDS population. They may also present with neurally mediated hypotension, orthostatic hypotension or orthostatic intolerance [12]. Patients usually complain of palpitations, lightheadedness, blurry vision, mental fog, and/or fatigue. On examination, patients with POTS have a sustained increase in heart rate of $\geq 30$ beats/minute ( $\geq 40$ beats/minutes in patients age 18 or younger) within 10 minutes of standing with an absence of orthostatic hypotension $(>20$ $\mathrm{mmHg}$ drop in systolic blood pressure) [12]. These patients are treated with oral (or occasionally intravenous) sodium chloride, electrolyte fluids, vasopressors (midodrine) and/or beta blockers [13] [14].

Mast Cell Activation Syndrome (MCAS) is another common comorbid condition in EDS [15] [16]. Patients with MCAS present with multisystemic symptoms related to inappropriate activation of mast cells and subsequent effects due to the release of mast cell mediators [17] [18]. Clinical presentation of MCAS includes skin abnormalities (rashes, hives), unexplained pruritus, flushing, diarrhea, abdominal cramping, anaphylaxis, peptic ulcer, osteoporosis, dermatographism, asthma, gastroparesis, and nausea [19]. It is important to note triggers for MCAS exacerbations. Some of the more common triggers are changes in temperature, stress, physical stimuli such as pressure or friction, fever, infection, invasive procedures (general anesthesia, surgery), heat, radiologic contrast media, and drugs (opioids, non-steroidal anti-inflammatory drugs, neuromuscular blocking drugs, and antibiotics) [20]. Treatment of MCAS includes minimizing exposure to any triggering agents, antihistamines (H1 and $\mathrm{H} 2$ receptor antagonists), and mast cell stabilizers such as sodium cromoglycate. In the perioperative setting, severe flares can be handled with histamine $\mathrm{H} 1$ and $\mathrm{H} 2$ receptor antagonists, glucocorticoids (methylprednisolone $10-250 \mathrm{mg}$ IV/IM or $2 \mathrm{mg} / \mathrm{kg}$ for pediatric patients), and benzodiazepines [21]. If administering diphenhydramine IV push, it should be given over at least five minutes as these patients may be hyperreactive to chemicals leached from IV tubing. Occasionally, a continuous infusion of diphenhydramine is required. Exacerbation of Mast Cell Activation Syndrome (MCAS) may also exacerbate symptoms of POTS [16].

Gastrointestinal complaints are very common in the EDS population. Dysmotility (usually gastroparesis) is common [22]. Patients often complain of abdominal fullness or bloating after eating a small portion of food, as well as alternating diarrhea and constipation [23]. These patients may need to be kept NPO longer and should be considered full stomach at all times. Due to the risk of hypovolemia, liberalizing NPO for liquids should be considered. Caution should be exercised while doing mask ventilation to minimize the risk of intestinal rupture. Gastroparesis, MCAS and POTS all may increase the risk of postoperative nausea and vomiting [24]. Nausea in EDS can be particularly difficult to treat as it may stem from numerous causes including gastrointestinal slowing, MCAS, 
POTS, craniocervical instability (CCI), or centrally mediated causes (Chiari malformation, intracranial hypertension, intracranial hypotension, spontaneous CSF leak) [25].

Opioids administered during the perioperative period may increase nausea and gastroparesis. Opioids, especially kappa opioid receptor agonists inhibit the release of antidiuretic hormone and oxytocin, leading to a diuretic effect. Opioids also cause release of histamine which can cause hypotension. Morphine can cause peripheral venous and arteriolar dilation, reduced peripheral resistance, and inhibition of baroreceptor reflexes. Opioids may increase orthostatic intolerance in this population. Fentanyl and sufentanil do not have a significant effect on hemodynamics partly because they do not release histamine [26]. Anecdotally, it has been noticed that patients with EDS have a weak response to the analgesic effects of opioids. In the preoperative evaluation it is helpful to elicit any history of response to opioids in the past or past surgeries.

Neuropathic pain often occurs in patients with EDS [27]. This can be due to Small Fiber Peripheral Neuropathy, Complex Regional Pain Syndrome, and Entrapment Neuropathy [5]. The patient may present with allodynia, dysesthesia, and hyperalgesia. The preoperative evaluation may reveal possible Central Sensitization. The limb or body part with neuropathic pain will need careful handling so as not to exacerbate pain [28]. Ketamine, an effective analgesic for neuropathic pain, can be considered for perioperative use in order to minimize opioid requirements thus lowering the risk of gastroparesis and histamine release.

Patients with EDS often have an abnormal response to lidocaine [29]. They may have local anesthetic resistance and/or delayed onset. Alternatively, they may have local anesthetic sensitivity or a prolonged effect. Inquiry into any previous experience with local anesthetics may provide useful information. Atypical reactions to lidocaine are the most commonly reported, however it is unknown if this population responds abnormally to other local anesthetic agents. Anecdotally, mepivicaine may be a better choice [30].

Neuraxial blocks may be challenging in the patient with EDS. The potential for unpredictable effects of lidocaine should be taken into consideration. Patients with EDS are at increased risk of developing post-dural puncture headaches (PDPH) [31]. Kyphosis or scoliosis is unlikely to affect the spread of the local anesthetic unless it is very severe, as the spinal changes are usually dynamic in EDS and limited to the cervical and thoracic regions.

Peripheral nerve blocks also present certain additional considerations in EDS patients. The duration and density of the nerve block may be less than anticipated due to local anesthetic resistance. Preexisting motor and sensory deficits should be sought and documented. Additionally, if special positioning of the patient is required (extreme head rotation, prone position etc.), joints at increased risk of dislocation/subluxation should be protected as much as possible [32].

EDS patients may also present with an increased risk of complications due to bleeding disorders and/or tissue fragility [33]. Patients with EDS may have bleeding disorders. Easy bruising is a common feature of all EDS subtypes. It is 
unclear if this is due to platelet dysfunction, capillary fragility or both. Although vascular fragility is a feature of vEDS and in the rare subtype dermatosparaxis type EDS (dEDS), it can be present to a certain degree in almost any subtype of EDS. Bleeding in EDS may be from a coagulopathy or structural fragility of the vascular tree and its supporting matrix [34]. Desmopressin (DDAVP) has been shown to reduce transfusion requirements in EDS [35] [36]. When given preoperatively it reduces complications of bleeding significantly. When handling tissues, surgeons should be aware of potential tissue fragility and increased risk of bleeding. Ligating or stapling vessels may be preferred over cauterization [37].

\section{Intraoperative}

Patients with POTS should have an intravenous line placed as early as possible in order to be preloaded with normal saline. The amount of normal saline administered should be based on their duration of NPO status as well as other comorbidities. These patients may present with hypovolemia and tachycardia. Typically, adult patients with POTS are administered 1 to 2 liters of normal saline preoperatively.

Monitoring should be maintained as per standardized guidelines. A non-invasive blood pressure cuff is unlikely to cause significant bruising although providing padding may help prevent cutaneous injury. Exposure to adhesives from tape and ECG leads may result in cutaneous and/or systemic reaction.

Patient positioning should take into consideration joints that have a history of recurrent subluxations and/or dislocations [38]. Extreme caution should be paid to range of motion. Maintain limbs less than their limit of range of motion to avoid overstretching ligaments and joint capsules [32].

Patients with EDS are prone to pneumothorax, cervical spine abnormalities and TMJ subluxation/dislocation. Caution with mask ventilation is recommended.

All patients with EDS should be considered candidates for fiberoptic intubation in order to minimize manipulation of the head and neck. The laxity of the spinal ligaments puts these patients at significant risk for CCI and/or atlantooccipital instability.

Endotracheal intubation should be performed with the smallest acceptable endotracheal tube and cuff pressures should be minimized. There has been one reported case of tracheal rupture after intubation [39]. Patients with gastroparesis should be considered full stomach at all times. In cases involving rapid sequence induction with cricoid pressure, pressure should be applied gently given that tracheomalacia is a common feature in EDS.

There are no specific contraindications to using any specific anesthetic agents. Caution must be used with agents that release histamine in patients with coexisting MCAS. Induction agents that produce minimal change in hemodynamics such as etomidate or ketamine may be preferred. Fentanyl, sufentanil or keta- 
mine are good analgesics to use because of their ability to maintain hemodynamic stability without releasing histamine. Consideration can be given to utilizing intravenous acetaminophen and/or ketorolac perioperatively in order to minimize opioid requirements. Anecdotally, many patients with EDS have an unpredictable response to a number of opioids. They may respond more consistently to hydromorphone. Intraoperative neuromuscular monitoring should take into consideration that myopathy is common in EDS.

POTS and other forms of dysautonomia are common in EDS patients. Close monitoring for hemodynamic changes is important. Hypotension is best managed with boluses of normal saline with or without small doses of vasopressors. There may be wide fluctuations in heart rate, usually with an elevated baseline heart rate. Vital signs may not be a reliable indicator of the depth of anesthesia, therefore consideration should be given to using processed EEG to monitor depth of anesthesia.

\section{Postoperative}

In the immediate postoperative period, concerns specific to patients with EDS include hemodynamic instability (most notably in patients with POTS), exacerbation of MCAS symptoms, and visceral rupture. Other general concerns include pain management, bleeding, nausea and vomiting. There should be a high suspicion for occult bleeding in patients who have undergone any procedure, even a relatively non-traumatic one such as an endoscopy. Patients with tracheomalacia may have airway compromise in the postoperative period and should be observed closely. Patients with neuropathic pain may respond well to intravenous ketamine in the immediate postoperative period. Acetaminophen may be a good option for postoperative pain.

In the event of an exacerbation of MCAS, treatment should be started as soon as possible. The administration of histamine $\mathrm{H} 1$ and $\mathrm{H} 2$ receptor antagonists, cromolyn (oral or nebulized), or benzodiazepines may be beneficial [18].

Patients who have undergone neuraxial block and spinal surgeries such as tethered cord lysis or decompression for Chiari malformation should be carefully screened for post-dural puncture headache [31]. Pain may be a result of a dislocated joint and repositioning may alleviate pain significantly thus decreasing the need for analgesics. Some spine surgeries require lying supine for an extended period of time which can pose additional challenges for postoperative pain management.

\section{EDS in Pregnancy}

Pregnancy in EDS presents its own unique set of challenges. Although all types of EDS may predispose the parturient to higher rates of preterm delivery, perineal lacerations and postpartum hemorrhage, patients with hypermobile EDS (hEDS) appear to pose little added risk in pregnancy [40]. EDS patients require special consideration due to the risk of uterine rupture, uterine incision dehis- 
cence, and postpartum hemorrhage [41].

Musculoskeletal complaints may increase in pregnancy due to hormonal influences increasing joint laxity, however some women report being less symptomatic during pregnancy. Pubic symphysis dysfunction as well as increased instability of the sacroiliac joints is important considerations for positioning of the pregnant patient. Hormonal changes may increase laxity of the TMJ and/or cervical spine making airway management even more challenging. Gastroparesis may increase the risk of aspiration even further in this population.

In addition to the routine evaluation of the perioperative pregnant patient, details of the patient's history regarding EDS subtype, bleeding diathesis, spinal pathology, arterial dissection, arterial rupture, spontaneous organ rupture, and effectiveness of local anesthesia should be elucidated. Special considerations for analgesia/anesthesia options include possible atypical response to local anesthetics and hemodynamic changes due to dysautonomia [42]. Early fluid loading may minimize hypotension.

One study revealed a risk of approximately one in five for intra and postpartum hemorrhage irrespective of mode of delivery in EDS patients. No life-threatening complications or technique related-complications (e.g. spinal hematoma, intubation difficulties or hypotension) occurred in his study [40].

\section{Vascular EDS in Pregnancy}

Rudd et al reported maternal mortality rates as high as $25 \%$ in vascular EDS (vEDS). (69) However, survival analysis from a 2014 study suggests that although the pregnancy related death rate is almost 300 times as high as the rate in the general US population, pregnancy in vEDS does not significantly affect overall survival [43].

Severe bleeding must always be anticipated. For cesarean delivery in vEDS, prophylaxis with DDAVP and/or tranexamic acid [35] should be considered in addition to crossmatching sufficient amounts of RBC and autotransfusion. The optimal choice for anesthesia and/or analgesia should be based on the individual patient's presentation.

In vEDS, there is no clear preferred mode of delivery. Vaginal delivery may be complicated by vascular or uterine rupture. Cesarean section (CS) poses risks of wound dehiscence. The prevalence of vEDS is low (1:100,000 - 1:200,000) [44] Anesthetic considerations in this population have been addressed primarily through case reports. Some reports strongly advise against neuraxial blockade in vEDS [43] (67) although there are several more recent case reports/case series of successful neuraxial anesthesia in patients with vEDS and other subtypes [45]. No relevant studies have been performed, however PDPH may occur with greater frequency due to tissue fragility.

In a study by Murray et al in 2014 [43], interviews with 39 women with vEDS demonstrated that $46 \%$ of deliveries were uncomplicated, $19 \%$ of deliveries were preterm, $10.5 \%$ had hemorrhage sufficient to require transfusion or extended 
hospital care, 9.2\% had arterial dissection/rupture, and $2.6 \%$ had uterine rupture. Five maternal deaths occurred in 76 deliveries (6.5\%) due to arterial dissection/rupture in 4 patients and complications related to dehiscence of a CS incision in one patient. Two fatal aortic ruptures occurred during labor and one occurred 7 days after an uncomplicated cesarean delivery. Nonfatal complications included antenatal coronary artery dissection, splenic artery dissection, multiple arterial dissections, uterine rupture and bladder/venous tears during CS. The diagnosis of vEDS was not established in $>85 \%$ of women until after delivery, therefore these complication rates reflect routine perioperative management [43].

\section{Conclusions}

Patients with EDS have a unique set of medical conditions that must be understood when presenting for surgery and anesthesia. Although there are 13 subtypes of EDS, with three subtypes being more prevalent, similar precautions need to be applied to all of these patients. Knowing which subtype the patient has is helpful but does not exclude risks unique to other subtypes. For example, a patient with hEDS may or may not be at risk for vascular fragility. A team approach with close communication is essential to optimize outcomes.

EDS patients are at risk of Craniocervical Instability and/or TMJ dysfunction, therefore special consideration should be given to performing endotracheal intubation utilizing a fiberoptic or video laryngoscopic approach. These patients may present with hypovolemia as a result of their dysautonomia requiring aggressive IV fluid management. Positioning may pose additional challenges due to the increased risk of joint dislocation and subluxation. Patients with MCAS may react unpredictably to various environmental factors. They may react to rapid temperature changes, extreme temperatures, medications, contact with fabrics and plastics and airborne substances.

Caution should be exercised with regional or neuraxial blocks due to an unpredictable response to local anesthetics as well as potential increased risk of complications secondary to tissue fragility. A detailed preoperative history and physical examination will help understand each patient's baseline condition and issues. Further studies are needed in order to better understand the effects of anesthesia and surgery in patients with EDS.

The limitations of this review are that it was based on compiling information from different sources on EDS and anesthesia. No specific studies have been done on the effect or techniques of anesthesia in patients with EDS. In the future, more direct studies will be needed to understand the pharmacokinetics and hemodynamic effects of anesthesia in EDS.

\section{Other Considerations}

There is a paucity of literature on the optimal surgical techniques with regards to safe handling of tissue in EDS. Cutaneous sutures should be left in place twice as 
long and additional fixation of adjacent skin with adhesive tape can help prevent stretching of the scar. Surgical wounds should be closed without tension, preferably in two layers. It is important to avoid tension on sutures in order to avoid risk of tissue tear. Multiple layers of sutures may be required. Deep sutures should be applied generously and in close proximity to each other. Allografts for bone and tendon may be preferred to avoid using weak connective tissue from autografts.

Patients with EDS are prone to spontaneous CSF leaks. There is also a higher incidence of structural neurological issues such as Chiari malformation, Craniocervical Instability, idiopathic intracranial hypertension and Tethered Cord Syndrome.

\section{Results}

Perioperative anesthetic care of patients with EDS requires a coordinated effort by all providers. The most common type of EDS is the Hypermobile type (hEDS). Patients with EDS present for surgical procedures frequently due to their tissue fragility, joint instability, vascular fragility, and association with neurosurgical co-existing conditions. In a preoperative history and physical examination, it is important to note which joints are prone to recurrent subluxations or dislocations. Patients with EDS can have Craniocervical instability (CCI) with related deformation of the brainstem and upper spinal cord, basilar invagination or ventral brainstem compression. They can also have Chiari malformation with restricted CSF flow, Idiopathic Intracranial Hypertension, C4-C5 osteochondrosis and mid-thoracic osteochondrosis. This population is also prone to Temporomandibular Joint Dysfunction (TMJ). Obstructive sleep apnea has been reported in patients with EDS. Rib subluxations are common in EDS and should be considered in the differential diagnosis of chest pain. Dysautonomia is a common comorbid condition in EDS Mast Cell Activation Syndrome (MCAS) is another common comorbid condition in EDS. Postural orthostatic tachycardia syndrome (POTS) is the most common type of dysautonomia in the EDS population. Dysmotility (usually gastroparesis) is common. Opioids administered during the perioperative period may increase nausea and gastroparesis. Neuropathic pain often occurs in patients with EDS. This can be due to Small Fiber Peripheral Neuropathy, Complex Regional Pain Syndrome, and Entrapment Neuropathy. Patients with EDS often have an abnormal response to lidocaine. They may have local anesthetic resistance and/or delayed onset. EDS patients may also present with an increased risk of complications due to bleeding disorders and/or tissue fragility.

Patients with POTS should have an intravenous line placed as early as possible in order to be preloaded with normal saline. Patient positioning should take into consideration joints that have a history of recurrent subluxations and/or dislocations. Patients with EDS are prone to pneumothorax, cervical spine abnormalities and TMJ subluxation/dislocation. Caution with mask ventilation is recommended. Endotracheal intubation should be performed with the smallest ac- 
ceptable endotracheal tube and cuff pressures should be minimized. There are no specific contraindications to using any specific anesthetic agents. Caution must be used with agents that release histamine in patients with coexisting MCAS. Close monitoring for hemodynamic changes is important. Hypotension is best managed with boluses of normal saline with or without small doses of vasopressors. In the immediate postoperative period, concerns specific to patients with EDS include hemodynamic instability (most notably in patients with POTS), exacerbation of MCAS symptoms, and visceral rupture. Other general concerns include pain management, bleeding, nausea and vomiting. Pregnancy in EDS presents its own unique set of challenges. EDS patients require special consideration due to the risk of uterine rupture, uterine incision dehiscence, and postpartum hemorrhage.

\section{Author Contributions}

Pradeep Chopra, MD and Linda Bluestein, MD had full access to all the data in the study and took responsibility for the integrity of the data and the accuracy of the data analysis. Pradeep Chopra, MD and Linda Bluestein, MD designed the study protocol. Drs. Pradeep Chopra and Dr. Linda Bluestein managed the literature searches and summaries of previous related work and wrote the first draft of the manuscript. Pradeep Chopra, MD and Linda Bluestein, MD provided revision for intellectual content and final approval of the manuscript.

\section{Conflicts of Interest}

All the authors have no conflicts of interest to report. None of the authors of the manuscript received any remuneration. Further, the authors have not received any reimbursement or honorarium in any other manner. The authors are not affiliated in any manner with any pharmaceutical industry. There was no external funding in the preparation of this manuscript. Each author certifies that he or she, or a member of his or her immediate family, has no commercial association (i.e., consultancies, stock ownership, equity interest, patent/licensing arrangements, etc.) that might pose a conflict of interest in connection with the submitted manuscript.

\section{References}

[1] Genetics Home Reference. U.S National Library of Medicine. https:/ghr.nlm.nih.gov/condition/ehlers-danlos-syndrome\#statistics

[2] Malfait, F., Francomano, C., Byers, P., et al. (2017) The 2017 International Classification of the Ehlers-Danlos Syndromes. American Journal of Medical Genetics Part C: Seminars in Medical Genetics, 175, 8-26.

[3] Wiesmann, T., Castori, M., Malfait, F. and Wulf, H. (2014) Recommendations for Anesthesia and Perioperative Management in Patients with Ehlers Danlos Syndrome(s). Orphanet Journal of Rare Diseases, 9, Article No. 109.

http://www.ojrd.com/content/9/1/109

https://doi.org/10.1186/s13023-014-0109-5 
[4] Pepin, M.G. and Byers, P.H. (1993) Ehlers-Danlos Syndrome Type IV. In: Pagon, R.A., Bird, T.D., Dolan, C.R., et al., Eds., GeneReviews, University of Washington, Seattle.

[5] Kuczkowski, K.M. (2005) Ehlers-Danlos Syndrome in the Parturient: An Uncommon Disorder-Common Dilemma in the Delivery Room. Archives of Gynecology and Obstetrics, 273, 60-62. https://doi.org/10.1007/s00404-004-0671-5

[6] Hirsch, C., John, M.T. and Stang, A. (2008) Association between Generalized Joint Hypermobility and Signs and Diagnoses of Temporomandibular Disorders. European Journal of Oral Sciences, 116, 525-530. https://doi.org/10.1111/j.1600-0722.2008.00581.x

[7] Norton, L.A. and Assael, L.A. (1997) Orthodontic and Temporomandibular Joint Considerations in Treatment of Patients with Ehlers-Danlos Syndrome. American Journal of Orthodontics and Dentofacial Orthopedics, 111, 75-84. https://doi.org/10.1016/S0889-5406(97)70305-6

[8] Abel, M.D. and Carrasco, L.R. (2006) Ehlers-Danlos Syndrome: Classifications, Oral Manifestations, and Dental Considerations. Oral Surgery, Oral Medicine, Oral Pathology, Oral Radiology, and Endodontology, 102, 582-590.

https://doi.org/10.1016/j.tripleo.2006.03.018

[9] Guilleminault, C., Primeau, M., Chiu, H.Y., Yuen, K.M., Leger, D. and Metlaine, A. (2013) Sleep Disordered Breathing in Ehlers-Danlos Syndrome: A Genetic Model of OSA. Chest, 144, 1503-1511. https://doi.org/10.1378/chest.13-0174

[10] De Wandele, I., Rombaut, L., Leybaert, L., et al. (2014) Dysautonomia and Its Underlying Mechanisms in the Hypermobility Type of Ehlers-Danlos Syndrome. Seminars in Arthritis and Rheumatism, 44, 93-100. https://doi.org/10.1016/j.semarthrit.2013.12.006

[11] Roma, M., Marden, C.L., De Wandele, I., Francomano, C.A. and Rowe, P.C. (2018) Postural Tachycardia Syndrome and Other Forms of Orthostatic Intolerance in Ehlers-Danlos Syndrome. Autonomic Neuroscience, 215, 89-96. https://doi.org/10.1016/j.autneu.2018.02.006

[12] Celletti, C., Camerota, F., Castori, M., et al. (2017) Orthostatic Intolerance and Postural Orthostatic Tachycardia Syndrome in Joint Hypermobility Syndrome/Ehlers-Danlos Syndrome, Hypermobility Type: Neurovegetative Dysregulation or Autonomic Failure? BioMed Research International, 2017, Article ID: 9161865. https://doi.org/10.1155/2017/9161865

[13] Gazit, Y., Nahir, A.M., Grahame, R., et al. (2003) Dysautonomia in the Joint Hypermobility Syndrome. The American Journal of Medicine, 115, 33-40. https://doi.org/10.1016/S0002-9343(03)00235-3

[14] Wallman, D., Weinberg, J. and Hohler, A.D. (2014) Ehlers-Danlos Syndrome and Postural Tachycardia Syndrome: A Relationship Study. Journal of the Neurological Sciences, 340, 99-102. https://doi.org/10.1016/j.jns.2014.03.002

[15] Bonamichi-Santos, R.L., Yoshimi-Kanamori, K., Giavina-Bianchi, P. and Aun, M.V. (2018) Association of Postural Tachycardia Syndrome and Ehlers-Danlos Syndrome with Mast Cell Activation Disorders. Immunology and Allergy Clinics of North America, 38, 497-504. https://doi.org/10.1016/j.iac.2018.04.004

[16] Cheung, I. and Vadas, P. (2015) A New Disease Cluster: Mast Cell Activation Syndrome, Postural Orthostatic Tachycardia Syndrome, and Ehlers-Danlos Syndrome. Journal of Allergy and Clinical Immunology, 135, AB65. https://doi.org/10.1016/j.jaci.2014.12.1146

[17] Hamilton, M.J., Hornick, J.L., Akin, C., Castells, M. and Greenberger, N.J. (2011) 
Mast Cell Activation Syndrome: A Newly Recognized Disorder with Systemic Clinical Manifestations. Journal of Allergy and Clinical Immunology, 128, 147-152. https://doi.org/10.1016/j.jaci.2011.04.037

[18] Afrin, L.B. and Molderings, G.J. (2014) A Concise, Practical Guide to Diagnostic Assessment for Mast Cell Activation Disease. World Journal of Hematology, 3, 1-17. https://doi.org/10.5315/wjh.v3.i1

[19] Seneviratne, S.L., Maitland, A. and Afrin, L. (2017) Mast Cell Disorders in Ehlers-Danlos Syndrome. American Journal of Medical Genetics Part C: Seminars in Medical Genetics, 175, 226-236. https://doi.org/10.1002/ajmg.c.31555

[20] Alvarez-Twose, I., Gonzalex de Olano, D., SanchezMunoz, L., Matito, A., Esteban-Lopez, M., Vega, A. and Escribano, L. (2010) Clinical, Biological, and Molecular Characteristics of Clonal Mast Cell Disorders Presenting with Systemic Mast Cell Activation Symptoms. Journal of Allergy and Clinical Immunology, 125, 1269-1278. https://doi.org/10.1016/j.jaci.2010.02.019

[21] Akin, C. (2014) Mast Cell Activation Disorders. The Journal of Allergy and Clinical Immunology: In Practice, 2, 252-257. https://doi.org/10.1016/j.jaip.2014.03.007

[22] Castori, M., Morlino, S., Pascolini, G., Blundo, C. and Grammatico, P. (2015) Gastrointestinal and Nutritional Issues in Joint Hypermobility Syndrome/Ehlers-Danlos Syndrome, Hypermobility Type. American Journal of Medical Genetics Part C (Seminars in Medical Genetics), 169, 54-75. https://doi.org/10.1002/ajmg.c.31431

[23] Dordoni, C., Ritelli, M., Venturini, M., et al. (2013) Recurring and Generalized Visceroptosis in Ehlers-Danlos Syndrome Hypermobility Type. American Journal of Medical Genetics Part A, 161, 1143-1147. https://doi.org/10.1002/ajmg.a.35825

[24] Farmer, A.D., Fikree, A. and Aziz, Q. (2014) Addressing the Confounding Role of Joint Hypermobility Syndrome and Gastrointestinal Involvement in Postural Orthostatic Tachycardia Syndrome. Clinical Autonomic Research, 24, 157-158. https://doi.org/10.1007/s10286-014-0239-9

[25] Henderson Sr., F.C., Austin, C., Benzel, E., Bolognese, P., et al. (2017) Neurological and Spinal Manifestations of the Ehlers-Danlos Syndromes. American Journal of Medical Genetics. Part C, Seminars in Medical Genetics, 175, 195-211. https://doi.org/10.1002/ajmg.c.31549

[26] Stanley, T.H. (2014) The Fentanyl Story. The Journal of Pain, 15, 1215-1226. https://doi.org/10.1016/j.jpain.2014.08.010

[27] Hamonet, C., Gompel, A., Raffray, Y., et al. (2014) Multiple Pains in Ehlers-Danlos Syndrome. Description and Proposal of a Therapy Protocol. Douleurs, 15, 264-277. https://doi.org/10.1016/j.douler.2014.09.003

[28] Cattalini, M., Khubchandani, R. and Cimaz, R. (2015) When Flexibility Is Not Necessarily a Virture: A Review of Hypermobility Syndromes and Chronic or Recurrent Musculoskeletal Pain in Children. Pediatric Rheumatology Online Journal, 13, 40. https://doi.org/10.1186/s12969-015-0039-3

[29] Chopra, P., Tinkle, B., Hamonet, C., et al. (2017) Pain Management in the Ehlers-Danlos Syndromes. American Journal of Medical Genetics. Part C, Seminars in Medical Genetics, 175, 212-219. https://doi.org/10.1002/ajmg.c.31554

[30] Ericson Jr., W.B. and Wolman, R. (2017) Orthopaedic Management of the Ehlers-Danlos Syndromes. American Journal of Medical Genetics. Part C, Seminars in Medical Genetics, 175, 188-194. https://doi.org/10.1002/ajmg.c.31551

[31] Schievink, W.I., Gordon, O.K. and Tourje, J. (2004) Connective Tissue Disorders with Spontaneous Spinal Cerebrospinal Fluid Leaks and Intracranial Hypotension: 
A Prospective Study. Neurosurgery, 54, 65-70. https://doi.org/10.1227/01.NEU.0000097200.18478.7B

[32] Ohashi, N., Furutani, K., Ishii, H. and Baba, H. (2012) Perioperative Brachial Plexus Injury Caused by Hyperabduction of the Upper Extremity in a Patient with Ehlers-Danlos Syndrome in the Prone Position Masui, 61, 626-628.

[33] Anstey, A., Mayne, K., Winter, M., Van de Pette, J. and Pope, F.M. (1991) Platelet and Coagulation Studies in Ehlers-Danlos Syndrome. British Journal of Dermatology, 125, 155-163. https://doi.org/10.1111/j.1365-2133.1991.tb06063.x

[34] De Paepe, A. and Malfait, F. (2004) Bleeding and Bruising in Patients with Ehlers-Danlos Syndrome and Other Collagen Vascular Disorders. British Journal of Haematology, 127, 491-500. https://doi.org/10.1111/j.1365-2141.2004.05220.x

[35] Stine, K.C. and Becton, D.L. (1997) DDAVP Therapy Controls Bleeding in Ehlers-Danlos Syndrome. Journal of Pediatric Hematology/Oncology, 19, 156-158. https://doi.org/10.1097/00043426-199703000-00012

[36] Mast, K.J., Nunes, M.E., Ruymann, F.B. and Kerlin, B.A. (2009) Desmopressin Responsiveness in Children with Ehlers-Danlos Syndrome Associated Bleeding Symptoms. British Journal of Haematology, 144, 230-233.

https://doi.org/10.1111/j.1365-2141.2008.07446.x

[37] Freeman, R.K., Swegle, J. and Sise, M.J. (1996) The Surgical Complications of Ehlers-Danlos Syndrome. The American Surgeon, 62, 869-873.

[38] Dolan, P., Sisko, F. and Riley, E. (1980) Anesthetic Considerations for Ehlers-Danlos Syndrome. Anesthesiology, 52, 266-269. https://doi.org/10.1097/00000542-198003000-00015

[39] Besselink-Lobanova, A., Maandag, N.J.G., Voermans, N.C., et al. (2010) Trachea Rupture in Tenascin-X Deficient Type Ehlers-Danlos Syndrome. Anesthesiology, 113, 746-749. https://doi.org/10.1097/ALN.0b013e3181e19c0f

[40] Castori, M., Morlino, S., Dordoni, C., et al. (2012) Gynecologic and Obstetric Implications of the Joint Hypermobility Syndrome (a.k.a. Ehlers-Danlos Syndrome Hypermobility Type) in 82 Italian Patients. American Journal of Medical Genetics, Part A, 158, 2176-2182. https://doi.org/10.1002/ajmg.a.35506

[41] Hurst, B.S., Lange, S.S., Kullstam, S.M., et al. (2014) Obstetric and Gynecologic Challenges in Women with Ehlers-Danlos Syndrome. Obstetrics \& Gynecology, 123, 506-513. https://doi.org/10.1097/AOG.0000000000000123

[42] Rowe, P.C., Barron, D.F., Calkins, H., Maumenee, I.H., Tong, P.Y. and Geraghty, M.T. (1999) Orthostatic Intolerance and Chronic Fatigue Syndrome Associated with Ehlers Danlos Syndrome. The Journal of Pediatrics, 135, 494-499. https://doi.org/10.1016/S0022-3476(99)70173-3

[43] Murray, M.L., Pepin, M., Peterson, S. and Byers, P.H. (2014) Pregnancy-Related Deaths and Complications in Women with Vascular Ehlers-Danlos Syndrome. Genetics in Medicine, 16, 874-880. https://doi.org/10.1038/gim.2014.53

[44] Volkov, N., Nisenblat, V., Ohel, G. and Gonen, R. (2007) Ehlers-Danlos Syndrome: Insights on Obstetric Aspects. Obstetrical \& Gynecological Survey, 62, 51-57. https://doi.org/10.1097/01.ogx.0000251027.32142.63

[45] Rivera-Alsina, M.E., Kwan, P., Zavisca, F.G., Hopkins, S. and Abouleish, E. (1984) Complications of the Ehlers-Danlos Syndrome in Pregnancy. A Case Report. The Journal of Reproductive Medicine, 29, 757-759. 


\section{List of Abbreviations}

AAI (Atlantoaxial Instability)

CCI (Craniocervical Instability)

DDAVP (Desmopressin)

EDS (Ehlers Danlos Syndrome)

cEDS (classic type EDS)

clEDS (classic-like type EDS)

cvEDS (cardiovascular type EDS)

dEDS (dermatosparaxis type EDS)

hEDS (hypermobile type EDS)

vEDS (vascular type EDS)

HSD (hypermobility spectrum disorder)

MCAS (Mast Cell Activation Syndrome)

PDPH (Post-Dural Puncture Headache)

POTS (Postural Orthostatic Tachycardia Syndrome)

TMJD (Temporomandibular joint dysfunction)

TMJ (Temporomandibular joint) 